\title{
Air-gap formation by snow melting
}

\author{
YASUAKI NOHGUCHI \\ Nagaoka Institute of Snow and Ice Studies, NIED, Suyoshi, Nagaoka, Niigata 940, Japan
}

\begin{abstract}
This paper describes theoretically the formation of air gaps at the lower boundary of a snow cover during basal melting. In general, initiation of the formation of air gaps is dependent on both the horizontal heterogeneity of the snowmelt rate and viscous deformation. From this point of view, we propose a dimensionless parameter $\xi$ (bridge-effect ratio) which is a function of the amplitude and wavelength of the heterogeneity of the snowmelt rate at the base, and the density, thickness and viscosity of the snow. This parameter expresses the heterogeneity of the normal stress at the base. We derive a necessary condition for air-gap formation in terms of the bridge-effect ratio, and show that a large amplitude, a small wavelength, a high density, a thin layer and/or high viscosities are favorable for air-gap formation.
\end{abstract}

\section{INTRODUCTION}

In snowy regions, roofs and roads are sometimes heated to melt and remove snow on them. In this method, air gaps are frequently formed at the lower boundary of the snow in contact with the roof or the road. As air gaps may influence the efficiency of snowmelt, to design a proper roof- or road-heating system it is of practical importance to understand the mechanism of air-gap formation.

The problem of air-gap formation due to snowmelt from the bottom is related both to deformation of snow and to snowmelt. Ôra (1958) has studied the deformation of snow caves, and Kubota and Kojima (1976) have experimentally studied heat transfer within such an air gap. However, the formation of air gaps from a state of a uniform snow without air gaps has not been sufficiently investigated until now.

In this paper, we propose simple models to describe air-gap formation from a horizontally uniform snow cover without air gaps. Using these models, we derive theoretically the conditions for air-gap formation and then discuss the roles of each parameter governing air-gap formation.

\section{GOVERNING EQUATIONS}

If the snow is not deformed, the heterogeneity of the horizontal distribution of melt rate at the base of the snow will cause air gaps. In general, however, snow is deformed by gravity, so such gaps tend to be closed by viscous deformation of the snow. Therefore, air-gap formation depends on both the heterogeneity of the horizontal distribution of snowmelt rate and viscous deformation of snow by gravity (Fig. 1).

First, in order to obtain the condition for air-gap formation, we calculate the normal stress at the bottom of the snow at the time when a horizontally uniform snow cover with no air gaps begins to be melted from the bottom with horizontally heterogeneous melt rate.

Consider a two-dimensional homogeneous snow layer (density, $\rho$, and thickness, $L$ ) without air gaps on a horizontal infinite plane (Fig. 2a). We call this a homogeneous snow-layer model. In general, however, the snow density increases with increasing depth. Therefore, we consider a more general model composed of a homogeneous lower snow layer and a soft upper layer, called a two-layer model (Fig. 2b).

We assume that the viscous deformation of the homogeneous lower layer is governed by the following equations for the $x$ components,

$$
\begin{gathered}
\partial \sigma_{x} / \partial x+\partial \tau_{y x} / \partial y=\rho g, \\
\sigma_{x}=\eta_{\mathrm{c}} \partial u / \partial x, \\
\tau_{y x}=\eta_{\mathrm{s}} \partial u / \partial y,
\end{gathered}
$$

where $u, g, \eta_{\mathrm{c}}$, and $\eta_{\mathrm{s}}$ are the $x$ components of displacement speed, gravitational acceleration, and viscous coefficients of compression and shear, respec-

a

b
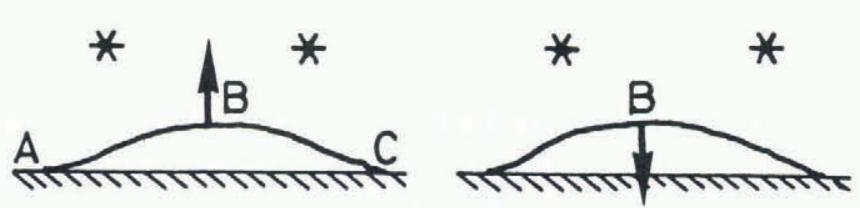

Fig. 1. Schematic representation of air-gap formation process: $a$, melting process; $b$, deforming process. 
tively. In this theory, for simplicity, the $y$ component of deformation is assumed to be negligibly small.

The boundary condition at the surface $(x=L)$ of the lower snow layer is

$$
\sigma_{x}=-w g
$$

where $w$ is weight per unit area of the upper snow layer. In particular, $w=0$ for the homogeneous snow-layer model.

Assuming snowmelt speed can be represented by the displacement speed of snow at the bottom, the heterogeneous boundary condition at the bottom $(x=0)$ of the snow is given by

$$
u=-u_{0}+\delta \sin k y
$$

where $u_{0}, \delta$, and $k$ are the horizontally averaged snowmelt speed, the amplitude of horizontal heterogeneity of snowmelt rate and its angular wave number, which is related to wavelength $\lambda$ as $2 \pi / \lambda$.

\section{SOLUTIONS}

If snow density, $\rho$, can be assumed to be constant, solutions of Equations (1)-(5) can be obtained (Nohguchi, 1992) in the form of the sum of homogeneous and heterogeneous components characterized by upper bar and $\Delta$, respectively.

$$
\begin{aligned}
u & =\bar{u}+\Delta u, \\
\bar{u} & =\frac{\rho g x^{2}}{2 \eta_{\mathrm{c}}}-\frac{H_{w} g x}{\eta_{\mathrm{c}}}-u_{o}, \\
\Delta u & =\delta \frac{\cosh \epsilon k(x-L)}{\cosh \epsilon k L} \sin k y, \\
\sigma_{x} & =\bar{\sigma}+\Delta \sigma, \\
\bar{\sigma} & =\rho g(x-L)-w g, \\
\Delta \sigma & =\delta \sqrt{\eta_{\mathrm{c}} \eta_{\mathrm{s}}} k \frac{\sinh \epsilon k(x-L)}{\cosh \epsilon k L} \sin k y, \\
\tau_{y x} & =\delta \eta_{\mathrm{s}} k \frac{\cosh \epsilon k(x-L)}{\cosh \epsilon k L} \cos k y,
\end{aligned}
$$

where

$$
\begin{aligned}
\epsilon & =\sqrt{\eta_{\mathrm{s}} / \eta_{\mathrm{c}}}, \\
H_{w} & =\rho L+w .
\end{aligned}
$$

Then, the normal stress at the bottom, $\sigma_{x 0}$, is represented by a horizontally homogeneous component, $\overline{\sigma_{0}}$, and a horizontally heterogeneous component, $\Delta \sigma_{0}$, as follows:

$$
\begin{aligned}
\sigma_{x 0} & =\overline{\sigma_{0}}+\Delta \sigma_{0}, \\
\overline{\sigma_{0}} & =-H_{w} g, \\
\Delta \sigma_{0} & =-\delta \sqrt{\eta_{\mathrm{c}} \eta_{\mathrm{s}}} k \tanh \epsilon k L \sin k y .
\end{aligned}
$$

These solutions are satisfied only for a state without air gaps and for a short time scale in which the given parameters can be considered constant.

\section{BRIDGE-EFFECT RATIO}

If the snowmelt rate is horizontally heterogeneous, then the normal stress at the bottom is also horizontally heterogeneous. Therefore, to express the heterogeneity of
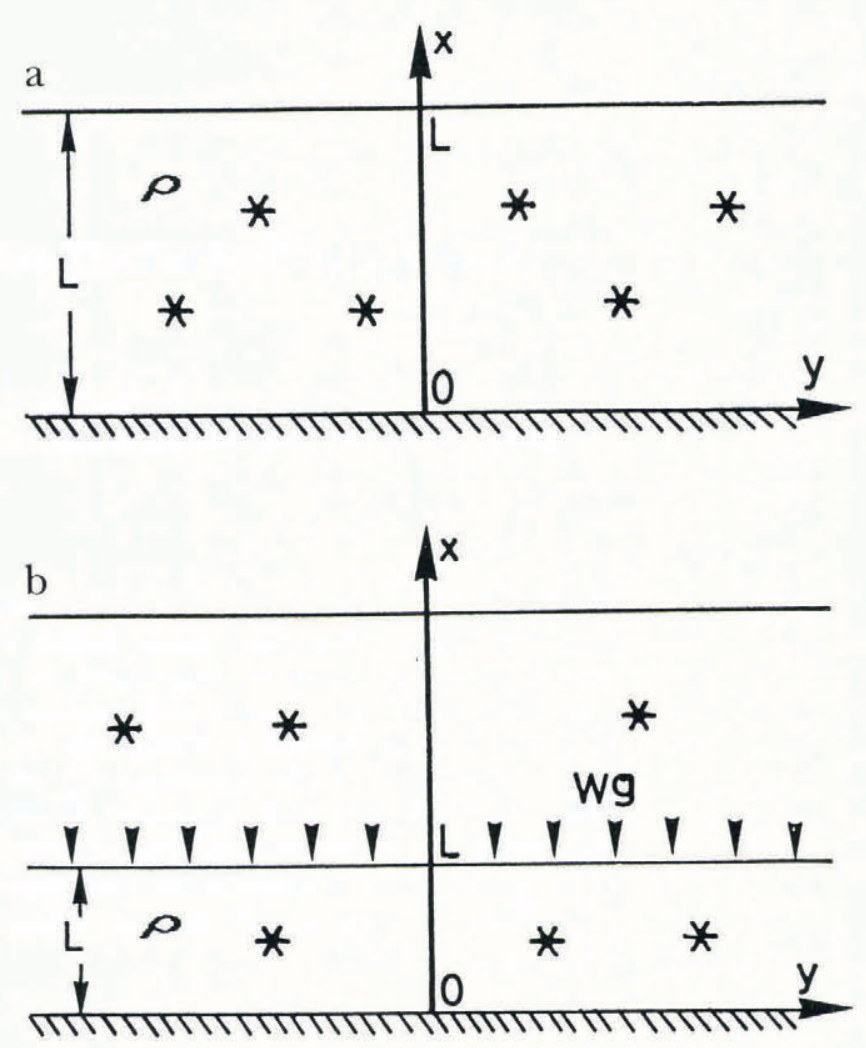

Fig. 2. Snow-cover models and coordinate system: $a$, homogeneous snow-layer model; $b$, two-layer model.

normal stress at the bottom due to that of snowmelt rate, we define a dimensionless parameter $\xi$, called the bridgeeffect ratio,

$$
\xi=\max \left(\Delta \sigma_{0}\right) /\left|\overline{\sigma_{0}}\right|=\frac{\sqrt{\eta_{\mathrm{c}} \eta_{\mathrm{s}}} k \tanh \epsilon k L}{H_{w} g} \delta .
$$

This dimensionless parameter represents the ratio of the maximum of the horizontally heterogeneous component of normal stress to the absolute value of the homogeneous component. Consequently, when $\xi=0$ the stress distribution at the bottom is uniform, and the heterogeneity of normal stress at the bottom increases with increasing bridge-effect ratio.

\section{CONDITION FOR AIR-GAP FORMATION}

When $\xi<1$ the normal stress at the bottom is everywhere negative, therefore, the bottom of the snow is everywhere in a compressive state. This means that air gaps cannot be formed because they are closed by viscous deformation. On the other hand, when $\xi>1$ there somewhere exists a position where the normal stress is positive, that is, under tension. In actual situations, a tensile state cannot exist unless the snow cover freezes to the bottom surface. In this case, air gaps can actually be formed by the heterogeneity of the snowmelt rate.

Thus, it is found that

$$
\xi<1
$$

is a sufficient condition for air gaps not to be formed,

$$
\xi>1
$$




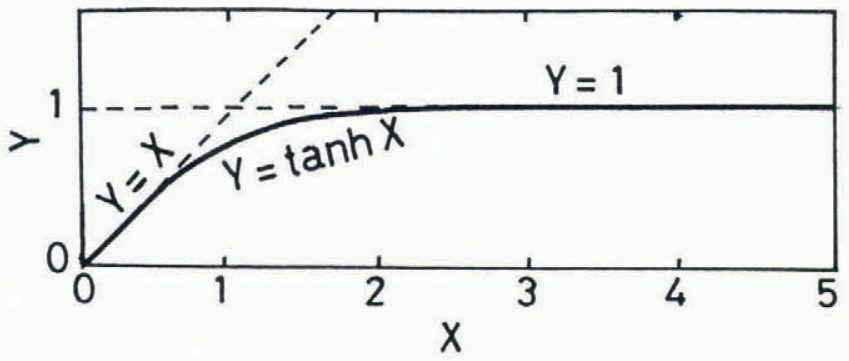

Fig.3. Approximations of a hyperbolic tangent function for long and short wavelengths.

is the necessary condition for their formation, and

$$
\xi=1
$$

is the critical condition.

\section{LONG-WAVE AND SHORT-WAVE APPROXIMATIONS}

The function tanh $\epsilon k L$ is shown in Figure 3. From this, when the wavelength, $\lambda$, of the heterogeneity of snowmelt speed is much longer than the thickness, $L$, of the snow layer, the bridge-effect ratio, $\xi$, can be approximately represented by

$$
\xi=\frac{\eta_{\mathrm{s}} k^{2} L}{H_{w} g} \delta
$$

We call this the long-wave approximation (LWA). Similarly, when the wavelength is much shorter than the thickness, $\xi$ can be approximately given as

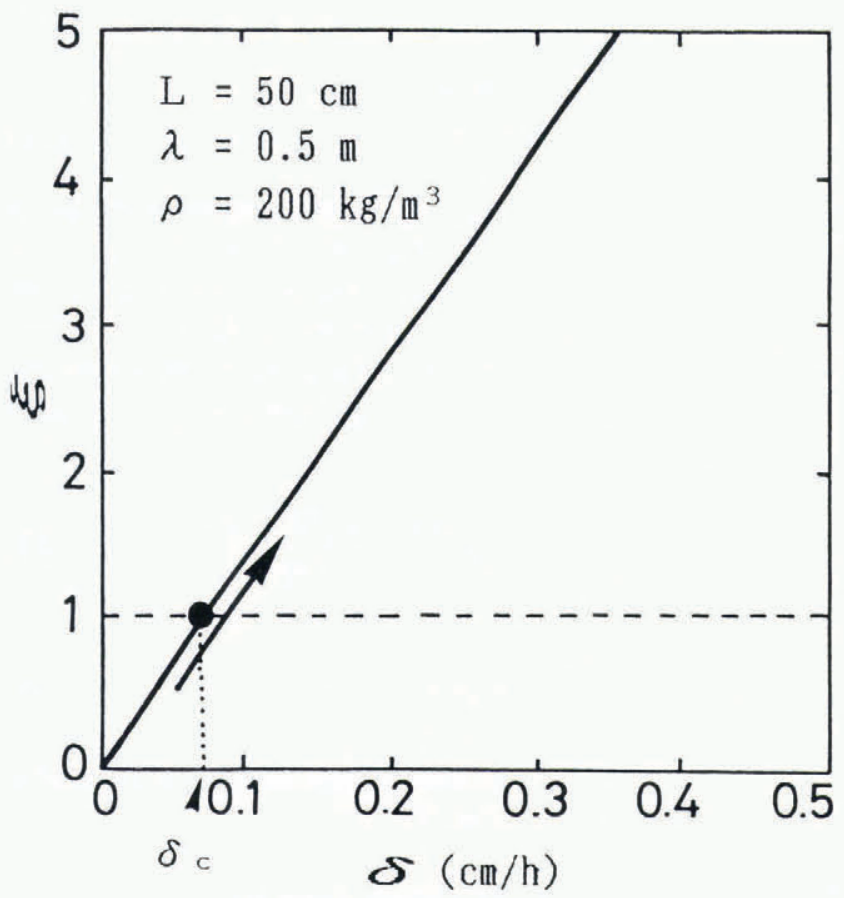

Fig. 4. Relation between bridge-effect ratios and amplitudes of heterogeneity of snowmelt speed in a homogeneous snow-layer mode. Viscosities are given by Shinojima (1967) as a function of snow density. The solid circle represents a critical point for air-gap formation.

$$
\xi=\frac{\sqrt{\eta_{\mathrm{c}} \eta_{\mathrm{s}}} k}{H_{w} g} \delta
$$

which we call the short-wave approximation (SWA).

For a homogeneous snow-layer model, $\xi$ reduces to

$$
\begin{aligned}
& \xi=\frac{\sqrt{\eta_{\mathrm{c}} \eta_{\mathrm{s}}} k \tanh \epsilon k L}{\rho g L} \delta=\xi_{0} \\
& \xi=\frac{\eta_{\mathrm{s}} k^{2}}{\rho g} \delta \quad \text { (LWA), } \\
& \xi=\frac{\sqrt{\eta_{\mathrm{c}} \eta_{\mathrm{s}}} k}{\rho g L} \delta \quad \text { (SWA). }
\end{aligned}
$$

\section{DISCUSSION}

\section{Effect of amplitude, $\delta$}

From Equation (18), we can see the bridge-effect ratio, $\xi$, is proportional to the amplitude of the heterogeneity of the snowmelt rate. $\xi$ increases with increasing amplitude, so a large amplitude is favorable for air-gap formation if the other parameters keep the same values (Fig. 4).

\section{Effect of wavelength, $\lambda$}

It is found that the bridge-effect ratio is an increasing function of the angular wave number from the following partial differential coefficient:

$$
\frac{\partial \xi}{\partial k}=\frac{\sqrt{\eta_{\mathrm{c}} \eta_{\mathrm{s}}} \delta}{H_{w} g}\left(\tanh \epsilon k L+\epsilon k L \operatorname{sech}^{2} \epsilon k L\right)>0 .
$$

Accordingly, the bridge-effect ratio decreases with decreasing wavelength, and so a small wavelength is

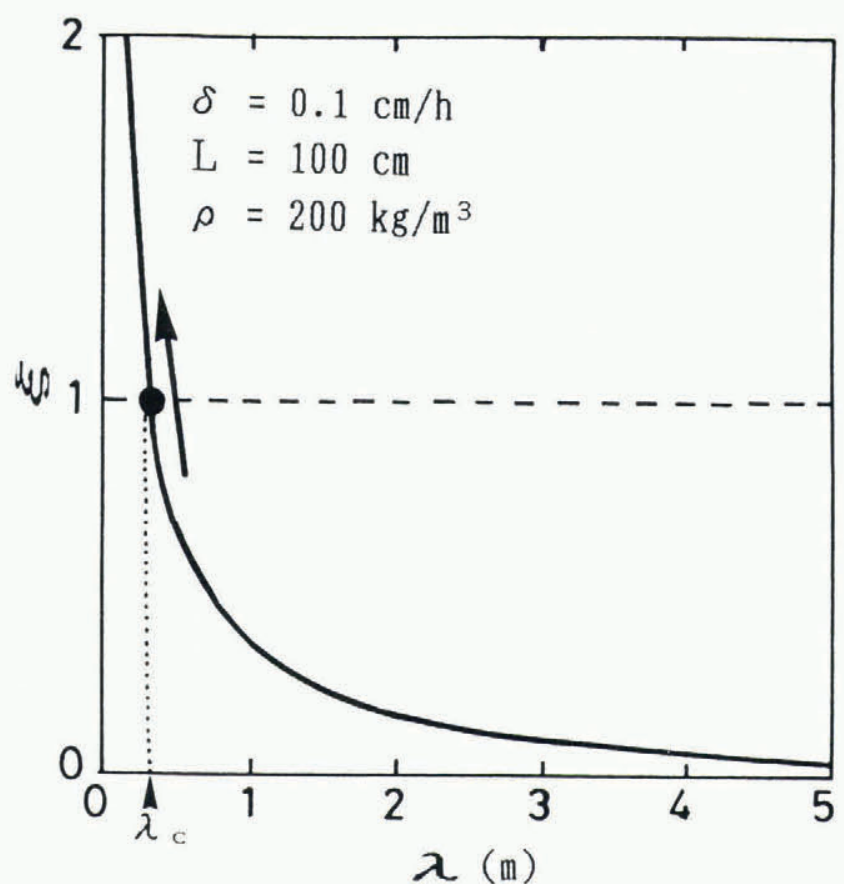

Fig. 5. Relation between bridge-effect ratios and wavelengths of heterogeneity of snowmelt speed in a homogeneous snow-layer mode. Viscosities are given by Shinojima (1967) as a function of snow density. The solid circle represents a critical point for air-gap formation. 
favorable for air-gap formation if the other parameters are the same (Fig. 5).

\section{Effect of snow thickness, $L$, in a homogeneous} snow-layer model

From Equations (23)-(25), the partial differential coefficient of $\xi$ by $L$ in a homogeneous snow-layer model is

$$
\begin{array}{ll}
\frac{\partial \xi}{\partial L}=\frac{\sqrt{\eta_{\mathrm{c}} \eta_{\mathrm{s}}} k \delta}{\rho g} \cdot \frac{\epsilon k L \operatorname{sech}^{2} \epsilon k L-\tanh \epsilon k L}{L^{2}}<0, & (\mathrm{LWA}), \\
\frac{\partial \xi}{\partial L}=0 & \text { (SWA). }
\end{array}
$$

This means that snow thickness does not affect air-gap formation in the long-wave approximation, but that in the short-wave approximation a thick snow cover is favorable for its formation (Fig. 6).

\section{Effect of snow density, $\rho$, in a homogeneous snow- layer model}

In general, viscosity of snow is an increasing function of snow density, therefore, the bridge-effect ratio is related to snow density not only directly through itself but also indirectly through the viscosity.

Shinojima (1967) and Kojima (1957, 1958, 1967) suggested a relation between the viscous coefficient and the snow density:

$$
\eta=a \exp (\alpha \rho)
$$

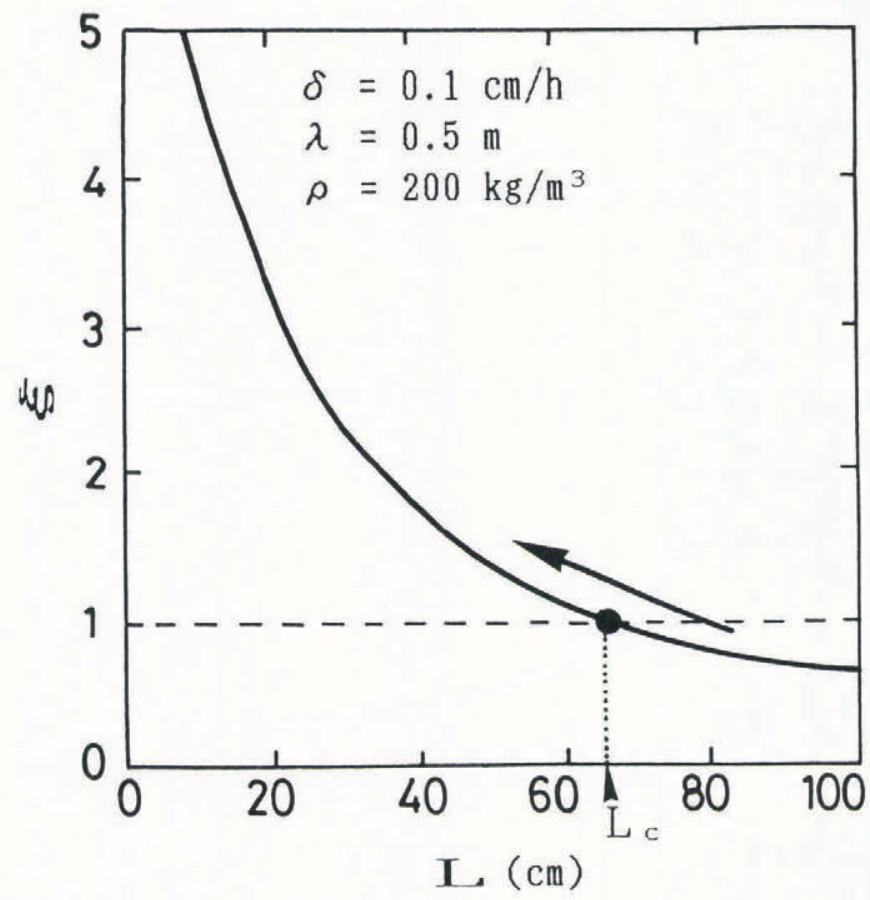

Fig. 6. Relation between bridge-effect ratios and snow thicknesses in a homogeneous snow-layer model. Viscosities are given by Shinojima (1967) as a function of snow density. The solid circle represents a critical point for airgap formation.

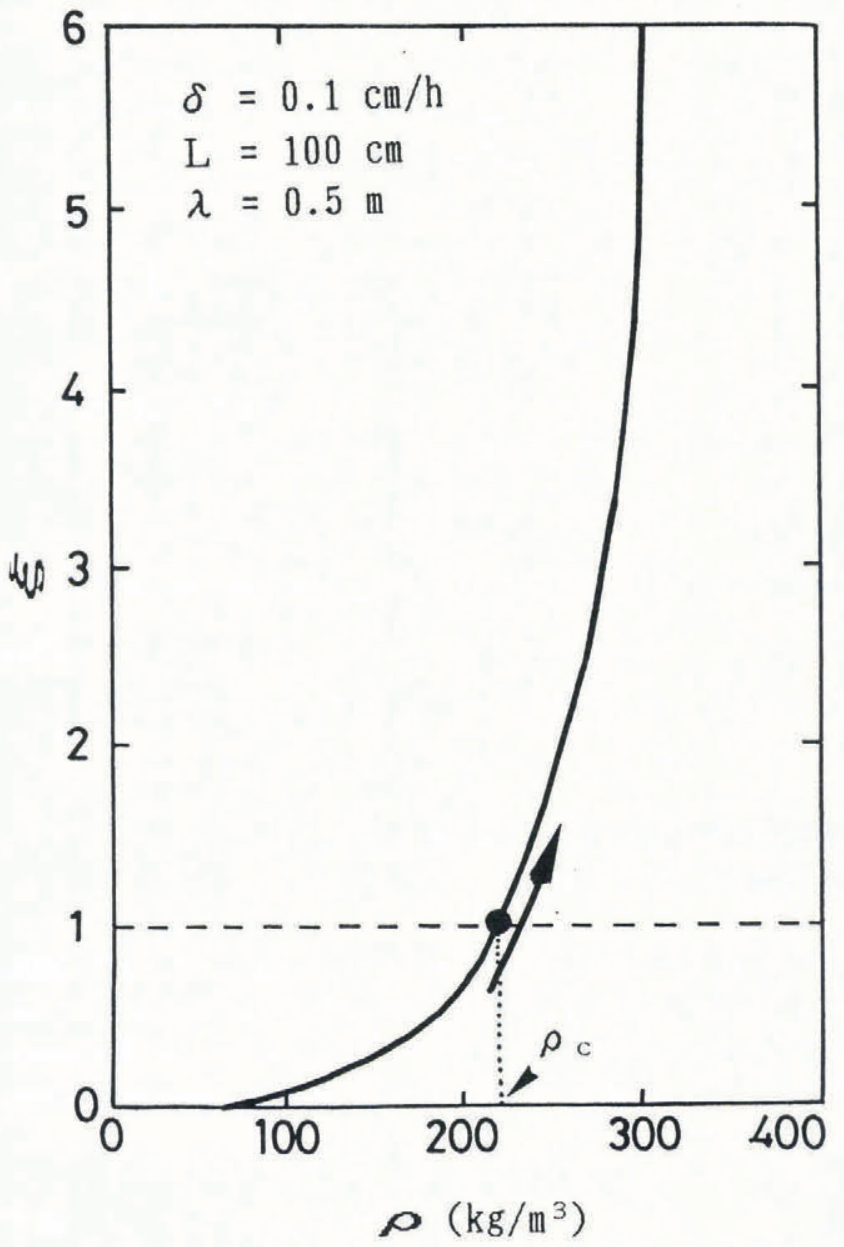

Fig. 7. Relation between bridge-effect ratios and snow densities in a homogeneous snow-layer model. Viscosities are given by Shinojima (1967) as a function of snow density. The solid circle represents a critical point for airgap formation.

Endo (1990) described it as

$$
\eta=a \rho^{4} \text {. }
$$

In either case, viscosity, $\eta$, is a strongly increasing function of $\rho$ for an ordinary value higher than $50 \mathrm{~kg} \mathrm{~m}^{-3}$ in comparison with snow density itself. Thus, the term related to snow density in Equation (23), $\sqrt{\eta_{\mathrm{c}} \eta_{\mathrm{s}}} / \rho$, becomes an increasing function of snow density.

Then, the partial differential coefficient of $\xi$ with respect to $\rho$ becomes

$$
\frac{\partial \xi}{\partial \rho}=\frac{\delta k \tanh \epsilon k L}{g L} \cdot \frac{\partial\left(\sqrt{\eta_{\mathrm{c}} \eta_{\mathrm{s}}} / \rho\right)}{\partial \rho}
$$

because

$$
\frac{\partial\left(\sqrt{\eta_{\mathrm{c}} \eta_{\mathrm{s}}} / \rho\right)}{\partial \rho}>0
$$

This result indicates that a high density is favorable for air-gap formation (Fig. 7).

\section{Effect of averaged snowmelt rate, $\boldsymbol{u}_{0}$}

The horizontal average of snowmelt rate is not directly included in Equation (18). This means that the homogeneous magnitude of snowmelt rate itself does not affect air-gap formation. However, if the amplitude of the 
heterogeneity of snowmelt rate were an increasing function of the average rate, then, from

$$
\frac{\partial \xi}{\partial u_{0}}=\frac{\partial \xi}{\partial \delta} \cdot \frac{\partial \delta}{\partial u_{0}}>0,
$$

a large average rate could be favorable for air-gap formation.

\section{Effect of the upper layer weight, $w$}

Equation (18) can be transformed into

$$
\frac{\xi}{\xi_{0}}=\left(\frac{W}{\rho L}+1\right)^{-1} .
$$

From this, it is found that the bridge-effect ratio decreases from $\xi_{0}$, which is the bridge-effect ratio only for a homogeneous lower snow layer, to zero as the weight of upper snow layer increases from zero to infinity (Fig. 8). This means that the addition of upper-layer load tends to close air gaps, even if the air-gap formation condition is satisfied for the lower snow layer.

\section{CRITIGAL VALUE OF EAGH PARAMETER}

When other parameters are fixed, the amplitude of heterogeneity of snowmelt rate which satisfies the critical condition of Equation (21) is

$$
\delta_{\mathrm{c}}=\frac{H_{w} g}{\sqrt{\eta_{\mathrm{c}} \eta_{\mathrm{s}}} k \tanh \epsilon k L},
$$

called the critical amplitude. Table 1 shows its values for a homogeneous snow-layer model. Then, the necessary condition for air-gap formation can be described also as

$$
\delta>\delta_{\mathrm{c}} .
$$

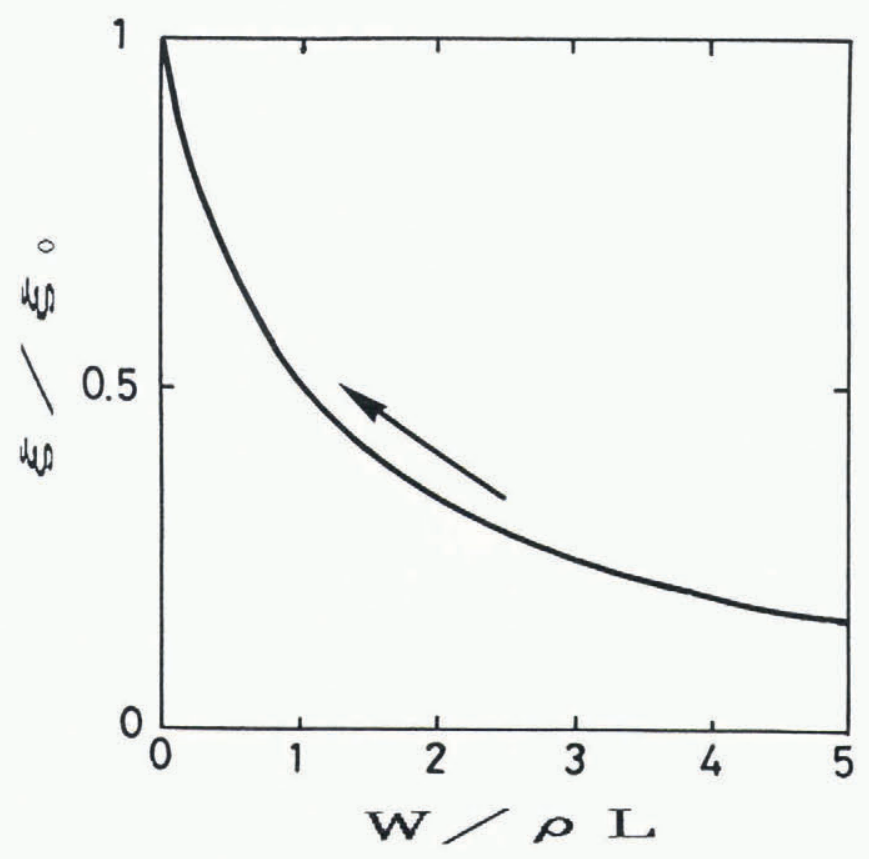

Fig. 8. Relation between bridge-effect ratios and upperlayer weights.
Table 1. Critical amplitude of heterogeneity of snowmelt

\begin{tabular}{|c|c|c|c|c|c|c|}
\hline \multirow{2}{*}{$\begin{array}{l}\text { Density } \\
\qquad(\rho)\end{array}$} & \multicolumn{6}{|c|}{$\begin{array}{l}\text { Length } \\
\text { (L) }\end{array}$} \\
\hline & $10 \mathrm{~cm}$ & $20 \mathrm{~cm}$ & $30 \mathrm{~cm}$ & $40 \mathrm{~cm}$ & $50 \mathrm{~cm}$ & $100 \mathrm{~cm}$ \\
\hline $\mathrm{kg} \mathrm{m}^{-3}$ & $\mathrm{~cm} \mathrm{~h}^{-1}$ & $\mathrm{~cm} \mathrm{~h}^{-1}$ & $\mathrm{~cm} \mathrm{~h}^{-1}$ & $\mathrm{~cm} \mathrm{~h}^{-1}$ & $\mathrm{~cm} \mathrm{~h}^{-1}$ & $\mathrm{~cm} \mathrm{~h}^{-1}$ \\
\hline
\end{tabular}
speed $\left(\delta_{\mathrm{c}}\right.$ in $\left.\mathrm{cm} \mathrm{h}^{-1}\right)$ for a homogeneous snow-layer model. Viscosities are given by Shinojima (1967) as a function of snow density

For $\lambda=0.25 \mathrm{~m}$

$\begin{array}{lllllll}100 & 0.05 & 0.094 & 0.14 & 0.19 & 0.24 & 0.47 \\ 200 & 0.0076 & 0.015 & 0.022 & 0.029 & 0.036 & 0.073 \\ 300 & 0.00093 & 0.0018 & 0.0027 & 0.0036 & 0.0045 & 0.009\end{array}$

For $\lambda=0.5 \mathrm{~m}$

$\begin{array}{lllllll}100 & 0.13 & 0.20 & 0.28 & 0.38 & 0.47 & 0.95\end{array}$

$\begin{array}{lllllll}200 & 0.021 & 0.031 & 0.044 & 0.058 & 0.073 & 0.15\end{array}$

$\begin{array}{lllllll}300 & 0.0026 & 0.0039 & 0.0054 & 0.0073 & 0.009 & 0.018\end{array}$

For $\lambda=1 \mathrm{~m}$

$\begin{array}{lllllll}100 & 0.47 & 0.54 & 0.65 & 0.79 & 0.97 & 1.9\end{array}$

$\begin{array}{lllllll}200 & 0.068 & 0.083 & 0.10 & 0.12 & 0.15 & 0.29\end{array}$

$\begin{array}{lllllll}300 & 0.0086 & 0.010 & 0.013 & 0.015 & 0.018 & 0.036\end{array}$

For $\lambda=2 \mathrm{~m}$

$\begin{array}{lllllll}100 & 1.7 & 1.8 & 1.9 & 2.1 & 2.3 & 3.9 \\ 200 & 0.27 & 0.28 & 0.30 & 0.33 & 0.36 & 0.60 \\ 300 & 0.033 & 0.034 & 0.037 & 0.041 & 0.045 & 0.071\end{array}$

For $\lambda=4 \mathrm{~m}$

$\begin{array}{lllllll}100 & 6.8 & 6.8 & 7.2 & 7.2 & 7.6 & 9.4\end{array}$

$\begin{array}{lllllll}200 & 1.1 & 1.1 & 1.1 & 1.1 & 1.2 & 1.4\end{array}$

$\begin{array}{lllllll}300 & 0.13 & 0.13 & 0.14 & 0.14 & 0.14 & 0.18\end{array}$

Table 2. Approximate representations of critical functions of each parameter for a homogeneous snow-layer model

\begin{tabular}{cccc}
\hline & $L W A$ & $S W A$ & $\begin{array}{c}\text { Air-gap formation } \\
\text { condition }\end{array}$ \\
\hline$\delta_{\mathrm{c}}$ & $\frac{\rho g}{\eta_{\mathrm{s}} k^{2}}$ & $\frac{\rho g L}{\sqrt{\eta_{\mathrm{c}} \eta_{\mathrm{s}}} k}$ & $\delta>\delta_{\mathrm{c}}$ \\
$L_{\mathrm{c}}$ & - & $\frac{\sqrt{\eta_{\mathrm{c}} \eta_{\mathrm{s}}} k \delta}{\rho g}$ & $L<L_{\mathrm{c}}$ \\
$k_{\mathrm{c}}$ & $\sqrt{\frac{\rho g}{\eta_{\mathrm{s}} \delta}}$ & $\frac{\rho g L}{\sqrt{\eta_{\mathrm{c}} \eta_{\mathrm{s}}} \delta}$ & $k>k_{\mathrm{c}}$ \\
$\lambda_{\mathrm{c}}$ & $2 \pi \sqrt{\frac{\eta_{\mathrm{s}} \delta}{\rho g}}$ & $\frac{2 \pi \sqrt{\eta_{\mathrm{c}} \eta_{\mathrm{s}}} \delta}{\rho g L}$ & $\lambda<\lambda_{\mathrm{c}}$ \\
\hline
\end{tabular}


Similarly, Table 2 summarizes the other critical parameters and the conditions for air-gap formation written in terms of the parameters.

\section{CONCLUDING REMARKS}

In this paper we derive theoretically the air-gap formation condition. This condition is described by the bridge-effect ratio as a function of the amplitude and wavelength of heterogeneity of snowmelt speed, snow density, snow thickness, viscosity of snow, and upper snow weight. As a result, we show that a large amplitude, a small wavelength, a high density, a thin layer, high viscosity, and/or a heavy upper snow are favorable for airgap formation.

\section{REFERENCES}

Endo, Y., Y. Ohzeki and S. Niwano. 1990. Relation between compressive viscosity and density of lowdensity snow. Seppyo. 7. Jpn. Soc. Snow Ice, 52(4), 267274. [In Japanese with English summary.]

Kojima, K. 1957. Viscous compression of natural snow layers III. Low Temp. Sci., Ser. A 17, 167-196. [In Japanese with English summary.]
Kojima, K. 1958. Viscous compression of natural snow layers IV. Low Temp. Sci., Ser. A 17, 53-64. [In Japanese with English summary.]

Kojima, K. 1967. Densification of seasonal snow cover. In Ôura, H., ed. Physics of snow and ice. Sapporo, Hokkaido University. Institute of Low Temperature Science, 929-952.

Kubota, H. and K. Kojima. 1976. Heat transfer in a vacancy formed below or within snow. Low Temp. Sci., Ser. A 34, 123-132. [In Japanese with English summary.]

Nohguchi, Y. 1992. Formation of air gaps in snow melting on a bottom boundary (I) - A theory for a homogeneous snow cover. Seppyo. 7. Jpn. Soc. Snow Ice, 54(2), 145-151. [In Japanese with English summary.]

Ôra, H. 1958. On the deformation of a snow cave. Low Temp. Sci., Ser. A 17, 65-79. [In Japanese with English summary.]

Shinojima, K. 1967. Study on the visco-elastic deformation of deposited snow. In Ôura, H., ed. Physics of snow and ice. Sapporo, Hokkaido University. Institute of Low Temperature Science, 875-907.

The accuracy of references in the text and in this list is the responsibility of the author, to whom queries should be addressed. 